\title{
Synergetic effect of green tea on polymer gel dosimeter and determination of optimal wavelength to choose light source for optical computed tomography
}

\author{
Sathiya Raj' ${ }^{1}$ James Jebaseelan Samuel${ }^{1}$, Senthil Kumar², Gopishankar Natanasabapathi ${ }^{3}$ \\ ${ }^{1}$ Division of Photonics, Nuclear and Medical Physics, School of Advanced Sciences, VIT University, Vellore, India \\ 2 Department of Physics, School of Basic Sciences, Vel Tech University, Avadi, Chennai, India \\ ${ }^{3}$ Department of Neurosciences, Gamma Knife unit, All India Institute of Medical Sciences, New Delhi, India
}

Received May 07, 2015; Revised December 08, 2015; Accepted December 15, 2015; Published Online December 20, 2015

\section{Original Article}

\begin{abstract}
Purpose: The ultimate aim of this study is to observe the effect of Green tea as a co-antioxidant in PAGAT gel dosimeter and evaluate the appropriate light source for scanning the PAGAT and NIPAM polymer gel. Methods: Both PAGAT (Poly Acrylamide Gelatin Tetrakis hydroxyl phosphonium chloride) and NIPAM (N-Isopropyl acrylamide) gel were prepared in normoxic condition. The green tea extract (GTE) was prepared and tested only on PAGAT. Co-60 teletherapy machine has been used for irradiation purpose, and the gel samples were scanned using UV-Visible spectrophotometer. Water equivalency of the gel has been tested in terms of their electron density, effective atomic number and Ratio of oxygen and hydrogen $(\mathrm{O} / \mathrm{H})$. We have used NIST XCOM database to test the water equivalency. Results: In this study we found that the GTE added to the gel do not respond to the given doses. By adding sugar we can enhance the sensitivity of the gel. Further investigations are required to use Green tea as a co antioxidant concentration of THPC (Tetrakis hydroxymethyl phosphonium chloride). The optimal wavelength with different region for scanning the PAGAT is 450 to $480 \mathrm{~nm}$ (Blue region), for NIPAM it is $540 \mathrm{~nm}$ and $570 \mathrm{~nm}$ (Green and yellow region). The PAGAT and NIPAM showed better sensitivity at $510 \mathrm{~nm}$. Both gels have their effective atomic number closer to water (NIPAM-7.2, PAGAT-7.379). Conclusion: As per our results, we concluded that GTE alone is not an effective co-antioxidant for polymer gels. When the GTE is combined with sugar and THPC, it protects the gel from pre-polymerization. This study strongly suggests that the blue light is an optimal source for scanning the PAGAT and green to yellow light for NIPAM gel. Though both gels were considered as water equivalent, the PAGAT is equivalent to water and the temporal stability of this gel is higher than NIPAM.
\end{abstract}

Keywords: PAGAT; NIPAM; GTE; NIST XCOM; THPC and Polymer Gel Dosimeter

\section{Introduction}

Verification of the 3D dose distribution is a part of patient specific quality assurance work in radiotherapy. Any departure from the accurate dose measurement makes a huge impact on treatment outcome. Polymer gel dosimeter offers reliable 3D dose verification. Since the gel is equivalent to water and it is sensitive to radiation, it can be used both as a phantom and radiation detector as well. It is known fact among the researchers that polymer gel dosimeter has initiated a new realm in the radiotherapy field due to its tissue equivalency, angular independency, good spatial resolution and also the availability of various readout techniques such as optical-computed tomography (CT), x-ray-CT, magnetic resonance imaging (MRI), ultrasound CT, and vibrational spectroscopy. However, the gel has its own disadvantages such as toxic chemical ingredients, time

Corresponding author: Sathiya Raj; Division of Photonics, Nuclear and Medical Physics, VIT-University, Vellore, India.

Cite this article as: Raj S, Jebaseelan Samuel J, Kumar S, Natanasabapathi G. Synergetic effect of green tea on polymer gel dosimeter and determination of optimal wavelength to choose light source for optical computed tomography. Int J Cancer Ther Oncol 2015; 4(1):412. D0I:10.14319/ijcto.41.2

[A part of this research was presented at IC3DDose Conference-2014, which was held from September 4-7, 2014 in Sweden] 
consumption for preparation, imaging procedure, and disposal. Among all these challenges, our focus is to introduce the natural component in gel dosimeter to make less use of toxic chemicals for preparation of gel. Many natural products having good antioxidant activity such as Acai berry, red berry, Green tea, turmeric, etc. ${ }^{1-3}$ Among these, green tea is easily available; it does not change the colour of gel and easy to prepare its extract. For these reasons, green tea was chosen for this study. It contain polyphenols were most of GTE polyphenols are flavonols known as catechin, which contains (+) catechin, (-) E picatechin, (-) E pigallocatechin, (-) Epicatechin, gallate, (-) Gallocatechingallate, and (-) Epigallocatechingallate etc. This catechin consumes the free radicals in water and protects the prepolymerization. Xuping et $a l^{4}$ reported glucose and urea which increases the sensitivity of polymer gel. Since common sugar is the disaccharide of glucose and fructose, a hypothesis has been made that sugar may increase the sensitivity of polymer gel. Optical-CT is one of the powerful tools to scan and extract the dose information from polymer gel. Many researchers utilized the Optical CT for reading the gel, which gives the reliable dose information on it. Utilization of X-ray CT and MRI is difficult in the busy hospitals. To get right dose information, the selection of light source is very important. To select an appropriate light source for polymer gel, we made a study based on the optical absorption property of the same. We chose PAGAT and NIPAM gel samples for this study.

\section{Methods and Materials}

In this study, we modified the recipe of PAGAT gel by introducing GTE and sugar. Initially, we tested the PAGAT gel by adding GTE and removed the THPC. The method of preparation of PAGAT has been reported in literature. ${ }^{5}$ The second PAGAT gel was tested by adding GTE, sugar and THPC. We have mentioned GTE added (without THPC) gel as PAG-GTE and GTE \& sugar added gel (with THPC) as PAGAT-GTS gel. For that, we prepared the GTE from dried green tea leaves and added into the PAG gel recipe. The preparation procedure of NIPAM has been done as per the report by Senden et al. ${ }^{6}$ Both the gels were irradiated by Co- 60 source and the dose information was extracted by UV-Visible spectrophotometer.

\subsection{Preparation of GTE}

Dried green tea leaves were commercially purchased. $1 \mathrm{~g}$ of leaves was soaked in $50 \mathrm{ml}$ of water (Millipore) for 4 hours. The solution was stirred in low RPM with a magnetic stirrer for 5 minutes. The solution was then heated up to 90 degree Celsius and finally kept in the room temperature for normal cool off.

\subsection{Preparation of PAG-GTE}

PAG-GTE was prepared from PAGAT recipe by replacing THPC with GTE. PAG-GTE has been prepared by adding green tea extract with different concentrations of
PAG-GTE-1 (0.6\%), PAG-GTE-2 (1\%) and PAG-GTE-3 $(0.23 \%+2.5$ gm of sugar $)$.

\subsection{Preparation of PAGAT-GTS}

PAGAT-GTS was prepared from PAGAT recipe with $0.23 \%$ of GTE and 2.5 gram of sugar without changing the concentration of THPC. After preparing the gel solutions, they were transferred to transparent plastic cuvettes, and sealed with parafilm in order to protect the entry of oxygen through the cuvette's opening. All the cuvettes were covered by black sheets to protect the photo-polymerization. Finally, the samples were kept at 4 degree Celsius for 24 hours before irradiation.

\subsection{Irradiation of Gel}

We assumed that the leakage radiation from the head of teletherapy machine can cause pre- polymerization. In order to achieve thermal equilibrium at room temperature, the samples were kept near the control console of the radiation treatment room. The angle of gantry, collimator and couch were set to zero. Gel cuvettes were placed on the $10 \mathrm{~cm}$ PMMA back scattering medium and the source to surface distance was set at $80 \mathrm{~cm}$ with the field size of $10 \times 10 \mathrm{~cm}^{2}$. We hypothesize that the laser can cause photo-polymerization hence isocenter matching of the sample with wall laser of therapy room was done immediately. The treatment time was calculated to deliver the dose at the centre of the cuvettes. Dose was delivered from 2 Gy to 12 Gy with an interval of 2 Gy for PAGAT and NIPAM. For PAG-GTE \& PAGAT-GTS, the dose delivered from 3 to 15 Gy with an interval of $3 G y$. All the exposed samples were transferred to the refrigerator to avoid the melting of gel due to the heat generated during irradiation. The temperature of the samples was maintained at 4 degree Celsius for 12 hours, allowing the samples to acquire complete polymerization.

\subsection{Analysis of dose response}

\subsubsection{Optical absorbance by spectrophotometer}

U2800-Hitachi was used for UV spectrophotometry on PAGAT and NIPAM. The un-irradiated gel ( 0 Gy) acted as a control sample for the irradiated one and it has been used for baseline correction in spectrophotometric analysis. The absorption wavelength was selected from $400 \mathrm{~nm}$ to $800 \mathrm{~nm}$ with $1 \mathrm{~nm}$ interval. The samples were read after a day of irradiation. The particle size was determined from the relation between maximum absorption and Mie-Debye's efficiency factor. It is given by the following equation,

$$
D=\frac{(K a) \lambda \max }{n \pi}
$$

Where, 'D' is the diameter of the particle, 'Ka' is the Mie-Debye efficiency factor which is found to be $4.34{ }^{7}$, ' $\mathbf{n}$ ' is the refractive index of the sample which is calculated as 1.5 . 


\subsubsection{Magnetic resonance imaging analysis}

MRI scan has been taken for both PAGAT and NIPAM gel to evaluate their dose response. MRI scan was performed after 1 day of irradiation. Gels were placed in MRI to reach the thermal equilibrium and the scan was performed after 2 hours. The scanning parameters for the gels are as follows: The field of view (FOV) - $250 \mathrm{~mm}$, pixel size $-0.49 \mathrm{~mm}$, slices thickness $-5 \mathrm{~mm}$ and number of echoes - 32. The time taken for scanning the gel was approximately 30 minutes. From the scan data we measured the pixel intensity and was normalized to 10 to the dose maximum.

\subsubsection{Elemental composition analysis}

In radiotherapy, dose measurements need to be performed in the water or water equivalent medium. Elemental composition analysis is the effective method to determine the water equivalency of any given medium. In this study, we used this method to evaluate the water equivalency of PAGAT and NIPAM. NIST (National Institute of Science and technology) XCOM database was used to perform this study.

\section{Results and Discussion}

The ratio between oxygen and hydrogen $(\mathrm{O} / \mathrm{H})$ in water is 7.9365 and for PAGAT, NIPAM, it is 7.17036 and 6.78853 respectively. The percentage difference $(0 / \mathrm{H})$ of PAGAT and NIPAM was $9.6 \%$ and $14.415 \%$ of water. Electron density and effective atomic number of both gels can be found by the following equations. ${ }^{8}$

$$
\rho e=\rho N A \cdot \sum \mathrm{i} W i\left(\frac{Z i}{A i}\right)
$$

Where, $\rho_{\mathrm{e}}$ is the electron density, $\boldsymbol{\rho}$ is density of the medium, NA is the Avogadro number (6.02 $\left.\times 10^{23}\right), W_{\mathrm{i}}$ is the fraction of weight of each element in gel, $\mathbf{Z}_{\mathbf{i}}$ is the Atomic weight and $\mathrm{Ai}$ is the Atomic number.

$$
Z_{\text {eff }}=\left(a_{1} Z_{1}{ }^{3.5}+a_{2} Z_{2}{ }^{3.5}+\ldots \ldots . . . a_{i} Z_{i}{ }^{3.5}\right)^{1 / 3.5}
$$

Where, $\mathbf{a}_{\mathbf{i}}$ is the fractional weight of $\mathrm{i}^{\mathrm{t}}$ element in gel and $\mathrm{Z}$ is the atomic weight of elements in gel. The electron density of the PAGAT, NIPAM and water was calculated as $3.4544 \times 10^{23} \mathrm{e} / \mathrm{cm}^{3}, 3.411 \times 10^{23} \mathrm{e} / \mathrm{cm}^{3}$ and $3.343 \times$ $10^{23} \mathrm{e} / \mathrm{cm}^{3}$ and the corresponding effective atomic number was 7.631, 7.200 and 7.417 respectively. Since the PAGAT and NIPAM have deviated by 2.8 and $2.93 \%$ from the effective atomic number of water, their electron density is found to be 3.3 and $2.0341 \%$ to that of water.

The following table shows the elemental composition of Water, PAGAT and NIPAM gel samples.
Table1: Elemental composition of Water, PAGAT and NIPAM.

\begin{tabular}{lcccccc}
\hline \hline \multicolumn{9}{c}{ Fractional weight of water, PAGAT and NIPAM medium } \\
\hline Medium & $\mathbf{W ( H )}$ & $\mathbf{W ( 0 )}$ & $\mathbf{W ( C )}$ & $\mathbf{W ( N )}$ & $\mathbf{W ( P )}$ & $\mathbf{W ( C L )}$ \\
\hline Water & 0.1119 & 0.8881 & - & - & - & - \\
PAGAT & 0.1061 & 0.7601 & 0.1032 & 0.0219 & 0.0002 & 0.0008 \\
NIPAM & 0.1064 & 0.7223 & 0.1364 & 0.0316 & 0.0015 & 0.0017 \\
\hline \hline
\end{tabular}

\subsection{UV Spectrophotometer analysis of PAGAT\& NIPAM}

Dose response of the NIPAM and PAGAT is as shown in Figures 1 \& 2. The graph was plotted for different wavelength and absorbance of both gels where, the absorbance found to increase with respect to the dose. PAGAT shows better linearity at $450 \mathrm{~nm}\left(\mathrm{R}^{2}=0.96064\right)$ and $480 \mathrm{~nm}\left(\mathrm{R}^{2}=0.97737\right)$ and for NIPAM it is in 510 $\mathrm{nm}\left(\mathrm{R}^{2}=0.9468\right), 540 \mathrm{~nm}\left(\mathrm{R}^{2}=0.97623\right)$ and $570 \mathrm{~nm}$ $(R 2=0.98932)$, respectively. To know the sensitivity of both the gels, the slope was extracted from dose response curve and plotted in Figure 3. Sensitivity of the NIPAM was found to be higher than PAGAT at 450 $\mathrm{nm}, 480 \mathrm{~nm}$ and $510 \mathrm{~nm}$. It was found that the sensitivity of both the gels was reduced beyond $510 \mathrm{~nm}$.

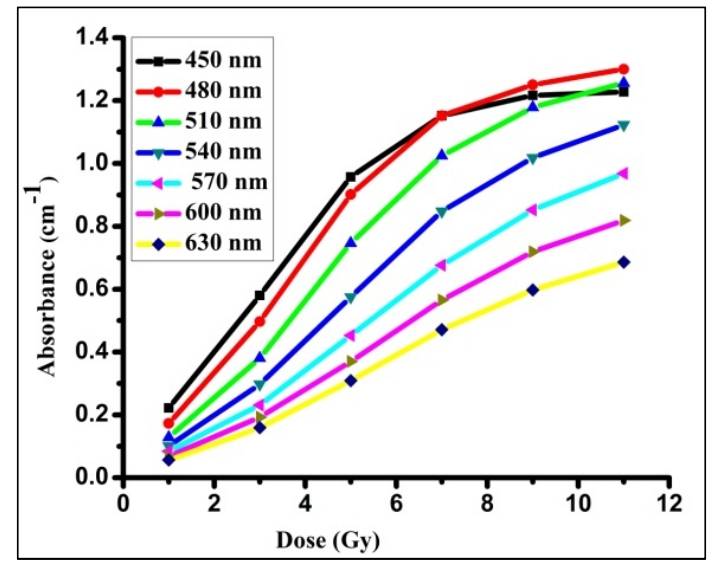

Figure 1: Dose response of NIPAM.

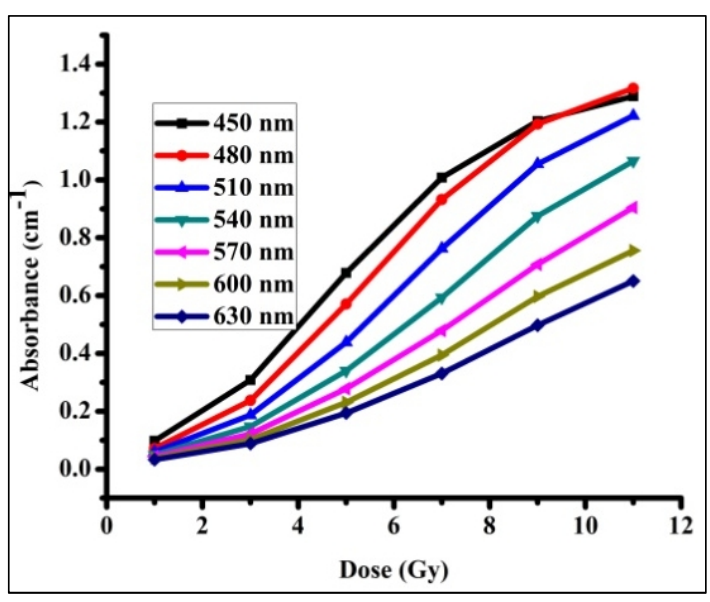

Figure 2: Dose response of and PAGAT gel. 


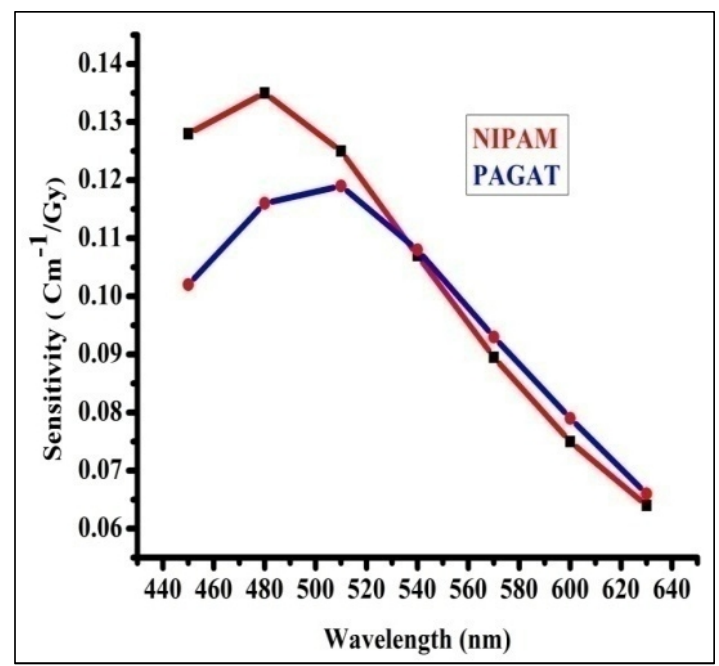

Figure 3: Sensitivity of NIPAM and PAGAT.

\subsection{MRI evaluation of dose response}

To evaluate the dose response of PAGAT and NIPAM, both gels were scanned and the graph was plotted between pixel intensity and dose. Figure 4 shows that both the gels are in good linearity. But, the NIPAM shows a low response to that of PAGAT. Dose responses of both the gels show a good linear function for both scanning modalities (Optical and Magnetic response).

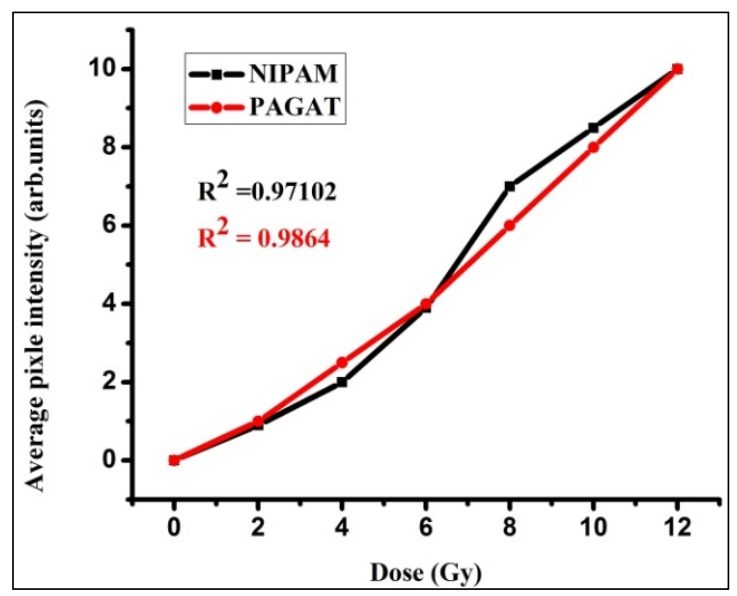

Figure 4: Dose response of NIPAM and PAGAT.

\subsection{Particle size analysis}

Particle size at different doses and time intervals are shown in Figures 5 and 6. It shows that the particle size increases with increasing dose.

The $\mathrm{R}^{2}$ values of PAGAT and NIPAM are almost same. Heather et al. ${ }^{7}$ and Maryanski et al. ${ }^{9}$ demonstrated the particle size increased with increasing dose. But our study found that the time intervals of post irradiation did not change the particle size significantly.

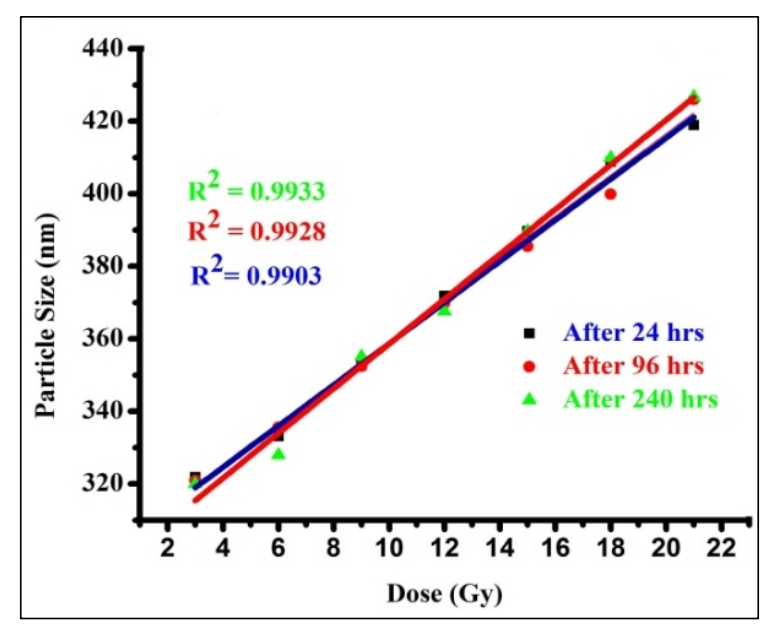

Figure 5: Particle size variation of PAGAT.

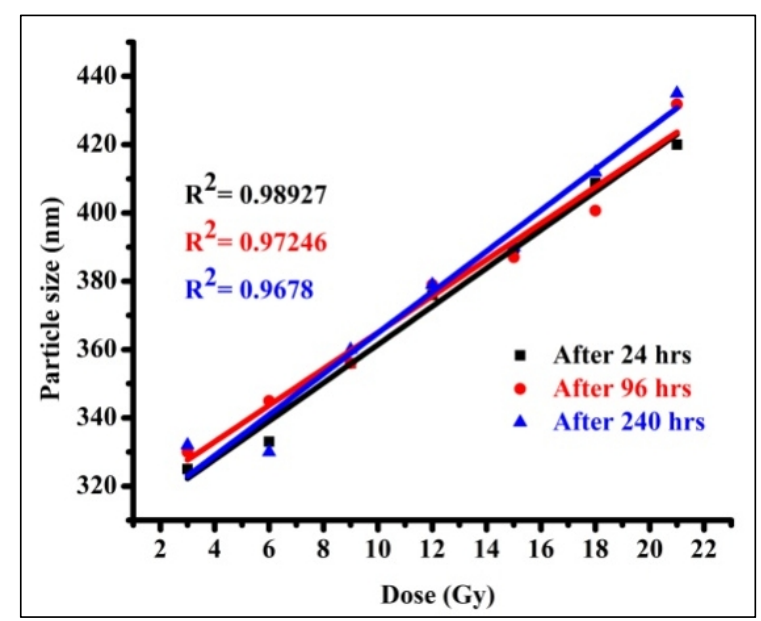

Figure 6: Particle size variation of NIPAM.

\subsection{Investigation of PAG-GTE and PAGAT-GTS}

One day after refrigeration, the samples were analyzed by double beam spectrophotometer. Un-irradiated gel samples acted as the baseline correction samples (control samples). The samples were read by spectrophotometer before irradiation. Figure 7 shows the response of PAG-GTE-1, PAG-GTE-2, PAG-GTE-3 and PAGAT-GTS. The PAG-GTE 1 \& 2 shows very less absorption whereas the PAG-GTE-3 shows a higher absorption than $1 \& 2$. PAG-GTE-3 contains GTE and sugar and this combination is the reason for a slight increase in the absorption.

But the absorption is not varied significantly with respect to the dose. Free radicals are the sources of polymerization and these free radicals are absorbed by GTE. Due to absorption of free radicals polymer chain formation has been reduced. The reduction of polymerization causes less absorbance peak for PAG-GTE $1 \& 2$. Both were almost overlapped in the dose response curve. In PAGAT-GTS, sugar and GTE were added and the concentration of THPC kept unchanged. GTE and sugar combination enhances the response of PAGAT-GTS than PAG-GTE 1, 2 \& 3. 


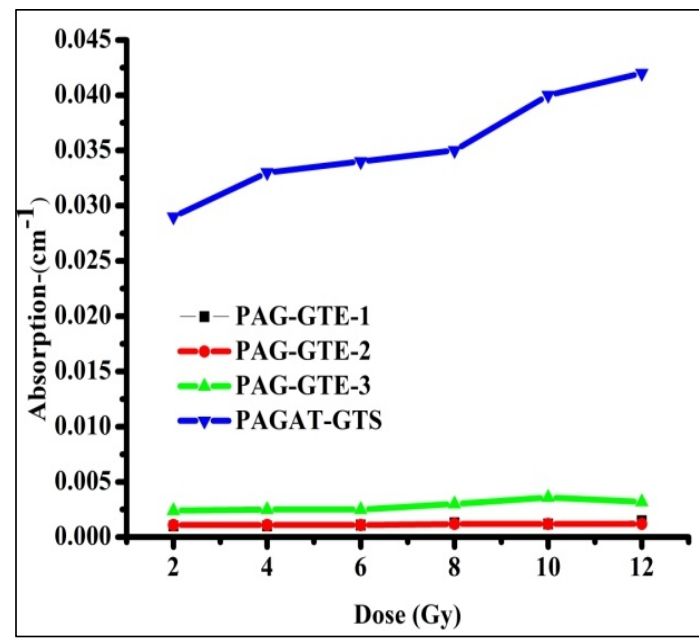

Figure 7: Dose response of PAGT-GTE 1, 2 \& 3 and PAGAT-GTS.

\subsection{Determination of optimal wavelength for Optical-CT}

Even though PAGAT and NIPAM have almost same elemental composition, they differ from the absorption property of light. PAGAT shows good linearity at 450 and $480 \mathrm{~nm}$ so the blue light source is optimal for scanning the PAGAT. For NIPAM, it is in 540 and $570 \mathrm{~nm}$ so green and yellow light is optimal. Further research is required to address the reason for changing the response of PAGAT and NIPAM for different wavelengths.

\section{Conclusion}

The general inference drawn from our investigation is that the response of the PAG-GTE is not valid for clinical application due to its poor absorption. But, if GTE is used with THPC as a co-antioxidant, it avoids pre-polymerization and enhance the response of the GTE based gel by adding sugar. This study suggests that the new composition of PAGAT-GTS gel can be used as a gel dosimeter. Further research is required to find out the optimal amount of the GTE without affecting the response of the polymer gel. To extract the dose information from Optical CT, blue light is an optimal for PAGAT, green and yellow light is optimal for NIPAM.

\section{Conflict of interest}

The authors declare that they have no conflicts of interest. The authors alone are responsible for the content and writing of the paper.

\section{References}

1. Kähkönen MP, Hopia AI, Heinonen M. Berry phenolics and their antioxidant activity. J Agric Food Chem. 2001;49:4076-82.
2. Huang WY, Lin YR, Ho RF, et al. Effects of water solutions on extracting green tea leaves. Scientific World Journal. 2013;2013:368350.

3. Durgaprasad S, Pai CG, Vaanthkumar, et al. A pilot study of the antioxidant effect of curcumin in tropical pancreatitis. Indian J Med Res. 2005;122:315-8.

4. Zhu X, Reese TG, Crowley EM, El Fakhri G. Improved MAGIC gel for higher sensitivity and elemental tissue equivalent 3D dosimetry. Med Phys. 2010;37:183-8.

5. Subramanian B, Ravindran PB, Baldock C. Optimization of the imaging protocol of an $\mathrm{X}$-ray CT scanner for evaluation of normoxic polymer gel dosimeters. J Med Phys. 2006;31:72-7.

6. Senden RJ, De Jean P, McAulley KB, Schreiner LJ. Polymer gel dosimeters with reduced toxicity: a preliminary investigation of the NMR and optical dose-response using different Monomers. Phys Med Biol. 2006; 51: 3301-14.

7. Whitney HM, Gore JC. Measurements of particle size in polymer gel dosimeter using spectrophotometry. J Phys.: Conf Ser. 2006; 56:160-3.

8. Khan FM. The physics of radiation therapy, $3^{\text {rd }}$ ed. Lippincott Williams and Wilkins Company; Philadelphia. 2003.

9. Maryanski MJ, Zastavker YZ, Gore JC. Radiation dose distribution in three dimensions from tomographic optical density scanning of polymer gels:II. Optical properties of the BANG polymer gel. Phys Med Biol. 1996; 41:2705-17. 